\title{
Research on Decision of Equipment maintenance and support task scheduling
}

\author{
Ma Yongfeng ${ }^{1, a}$,Qi Fengjun ${ }^{2, b}$,Wang Zhaojian ${ }^{3, c}$, Jiang Xishuang ${ }^{4, d}$, Liu Junjie ${ }^{5, e}$ \\ ${ }^{1}$ No. 1 Garden Road, Changchun City, Jilin Province, China \\ ${ }^{2}$ No. 1 Garden Road, Changchun City, Jilin Province, China \\ ${ }^{3}$ No. 1 Garden Road, Changchun City, Jilin Province, China \\ ${ }^{4}$ No. 1 Garden Road, Changchun City, Jilin Province, China \\ ${ }^{5}$ No. 1 Garden Road, Changchun City, Jilin Province, China

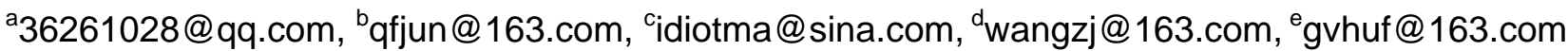

Keywords: Equipment maintenance, scheduling, algorithm

\begin{abstract}
By the analysis of maintenance scheduling problems, considering the battle effectiveness with multi-objects, aiming at such as defects only one for the current objects of the study, but does not consider sophistication of weapons, configuration and so on.The dual objects of the weighted operational time after the repair and the least numbers of delay are studied. The scheduling algorithm are given. Making up for these shortcomings, the scheduling is more reasonable. Finally, an example shows that the method of solving the model is simple, and there to be shorter computing time. The maintenance task scheduling method is valid.
\end{abstract}

\section{Introduction}

The equipment is an important part of the combat effectiveness of the armed forces, and equipment maintenance is an important factors of maintenance, restores and even improving the combat effectiveness. The battle damaged equipment scientific and rational way for quick repairs, is not only an important factor of the recovery equipment combat unit integrity, but also directly affect the fast, high-strength weaponry combat unit deployed ${ }^{[1]}$. In recent years, the problem of equipment maintenance and support have been more extensive researched, especially to target maintenance effectiveness, combat time after repair ${ }^{[3,4]}$. However, the following deficiencies exist in the current research:

First, considering the target is too single. Existing research mainly consider the contribution to the combat effectiveness of the combat time after repair, the shorter stay in maintenance system of the malfunction equipments, the longer into combat, the greater contribution to the combat effectiveness;

Second, there were not be taken into account the factors associated closely with the combat effectiveness, for example, the degree of advanced equipment, configuration, importance and so on;

Third, the existing algorithms are no strict argument or description of their complexity, these algorithms may not be valid algorithm.

In view of these circumstances, we will consider delays as little as possible under the optimal premise of the longest combat time after repair (the shortest stay in maintenance system of the malfunction equipments), consider the degree of advanced equipment, configuration factors (ie, weight), also establish a mathematical model with an effective algorithm to achieve a more effective maintenance task scheduling.

\section{The Analysis Of Maintenance Scheduling Factors}

1.1 Maintenance tasks assignation must be measured up by combat units. The most prominent feature of modern warfare is the confrontation between equipment's systems, only matching weapon equipment can produce more strong combat effectiveness. Therefore, when multiple 
weapons and equipment are broke down, we must repair completely in order to make the most powerful comat effectiveness, so maintenance tasks assignation must be measured up by combat units. combat unit refer to the unit that can be operations and training independently, generally be made up of weapons systems ,supporting resources and users. Weapon system consists of multiple combat equipment, a lot of malfunction equipment will appear, malfunction equipment form maintenance tasks, maintenance scheduling is responsible for the coordination, control of maintenance tasks and maintenance resources optimization ${ }^{[4]}$.

1.2 Maintenance process. in the process of equipment maintenance scheduling, variety of professionals and damaged equipments work in turn. maintenance process is composed of fault identification, fault location, troubleshooting and assembly debugging, the following maintenance activities can not be carried out until the former was done, completion these maintenance activities require a corresponding set of person, equipment, premises and maintenance resources, that are seen as a maintenance team.

1.3 The analysis of combat effectiveness factors. Combat effectiveness is related to many factors, wherein the "battle time" is a very important factor, once the equipments breakdown, they should be repaired, the shorter maintenance time the stronger combat effectiveness. But, different equipment impact on combat effectiveness, the more advanced equipment, the more contribution to combat effectiveness $^{[8]}$. In addition, frequent wartime damage and relatively limited maintenance capacity cause delays inevitable. If we can guarantee under the premise of "the shortest time in maintenance system ", the number of delayed equipment is as small as possible, which can increase the amount of equipment into battle and improve the combat effectiveness. Therefore, considering these factors, maintenance task scheduling has a very important research value.

\section{Model Establishment}

\subsection{Basic assumptions}

First, consider only one maintenance team; second, the maintenance procedure can not be interrupted; third, the maintenance team only repair one equipment at any time.

2.2 Analysis of the objective function

2.2.1 Maximization of combat time after repair

Assuming that the over time of fighting is $T$, there are $n$ combat units, unit $i(i=1,2, \cdots, n)$ have $n_{i}$ equipments, equipment $j$ of unit $i$ is recorded as $t_{i j}$, average maintenance time of $t_{i j}$ is $p_{i j}$, the weight of the combat unit $i(i=1,2, \cdots, n)$ is $w_{i}$, all malfunction equipment maintenance time of the combat unit $i$ is $c_{i}$, maintenance required time is $p_{i}$, started time is $s_{i}$, $c_{i}=s_{i}+p_{i}$, maintenance time of $t_{i j}$ is $c_{i j}$. Special agreement on unit $k$ does not requires maintenance, $c_{k}=0, c_{k j}=0\left(j=1,2, \cdots, n_{k}\right)$, when equipment $j$ of unit $k$ does not requires maintenance, $c_{k j}=0, j \in\left\{1,2, \cdots, n_{k}\right\}$.

First, only consider the contribution to the combat effectiveness of combat time after the repair, maintenance tasks assigned objective function is:

$$
\max \sum_{i=1}^{n}\left(T-c_{i}\right)=\max \left(\sum_{i=1}^{n} T-\sum_{i=1}^{n} c_{i}\right)=\max \left(T-\sum_{i=1}^{n} c_{i}\right)=T-\min \sum_{i=1}^{n} c_{i}
$$

The objective function is transformed into: $\min \sum_{i=1}^{n} c_{i}, c_{i}=\max _{1 \leq j \leq n_{i}} c_{i j}{ }^{[5]}$.

Second, consider the contribution to the combat effectiveness of combat time after repair and the equipment advanced degree, the objective function is:

$$
\max \sum_{i=1}^{n} w_{i}\left(T-c_{i}\right)=\max \left(\sum_{i=1}^{n} w_{i} T-\sum_{i=1}^{n} w_{i} C_{i}\right)=\max \left(T-\sum_{i=1}^{n} w_{i} c_{i}\right)=T-\min \sum_{i=1}^{n} w_{i} c_{i}
$$


The objective function is transformed into: $\min \sum_{i=1}^{n} w_{i} c_{i}, \quad c_{i}=\max _{1 \leq j \leq n_{i}} c_{i j}{ }^{[5]}$.

\subsubsection{The number of combat units delay minimization}

The situation in wartime is complex and changing, damaged weapons become more frequent, in the process of maintenance, we need a lot of resources, a lot of equipment to be repaired within a relatively short time Cause delayed inevitably. In certain circumstances, the length of the delay is not important, as long as there is delay occurs, the consequences are the same. Therefore, under the optimal premise to ensure the main objective, minimization the delayed units is very important to combat effectiveness.

Assume that all breakdown equipment's actual maintenance time of unit $i$ is $c_{i}$, maintenance required time is $p_{i}$, started time is $s_{i}, c_{i}=s_{i}+p_{i}$. All fault equipment maintenance time required by higher level is $d_{i}$. If $c_{i}>d_{i}$, then delayed occur, recorded as $u_{i}=1$,if $c_{i} \leq d_{i}$, then delayed dose not occur, recorded as $u_{i}=0$. So the goal is minimization the total number of delayed equipment $\sum u_{i}$.

2.2.3 Under the premise of maximization of combat time after repair, minimize the number of delayed combat units

There are many factors related to the combat effectiveness in maintenance support, but, each of them have different contribution to combat effectiveness. So, we select the important factors to be studied, these factors form the objective function of the maintenance scheduling, this is a multi-objective maintenance decision problem. Between these objective, it is difficult to achieve Optimal at the same time. So in the case of main objective have been achieved optimal, we make another objective better. So the objective function we mainly research are $\sum u_{i} / \sum_{i=1}^{n} c_{i}$ and $\sum u_{i} / \sum_{i=1}^{n} w_{i} c_{i}$

\section{Analysis Of Algorithm}

3.1 Optimal single-objective maintenance order

Sort, is allocation of time to complete some tasks and achieving optimal objective. It play a auxiliary scientific decision-making role to improving performance, the development and deployment of resources, progressing arrangements ${ }^{[7]}$.

Lemma 1: malfunction equipment's maintenance time of combat unit $i$ is $p_{i}(i=1,2, \cdots, n)$, the corresponding weights is $w_{i}$, to the objective $\min \sum_{i=1}^{n} w_{i} c_{i}$, optimal maintenance order is non-increasing sequence $\frac{w_{i}}{p_{i}}$ (WSPT scheduling algorithm $)^{[2]}$

Lemma 2: under the case of single maintenance team, all malfunction equipment's maintenance time of combat unit $i(i=1,2, \cdots, n)$ is $p_{i}$, to the objective $\min \sum_{i=1}^{n} c_{i}$, optimal maintenance order is from small to large according to maintenance time (SPT scheduling algorithm) ${ }^{[2]}$.

Lemma 3: under the case of single maintenance team, to the objective $\min \sum u_{i}$, optimal maintenance order of combat unit $i(i=1,2, \cdots, n)$ is Unabated order about duration $d_{i}$ ( Moore-Hodgson scheduling algorithm) ${ }^{[2]}$.

3.2 Optimal multi-objective maintenance order

The problem of minimize the number of delayed combat units under the premise of maximize of 
combat time after repair is the Objective of $\min \sum u_{i}$ (or $\min \sum_{i=1}^{n} w_{i} c_{i}$ ) optimal, the problem of optimal objective $\min \sum u_{i}$, recorded as $\sum u_{i} / \sum_{i=1}^{n} c_{i}$, can be solved by Moore-Hodgson/SPT algorithm, its complexity is $O(n \log n)^{[6]}$. To $\sum u_{i} / \sum_{i=1}^{n} w_{i} c_{i}$, it can be solved by Moore-Hodgson/WSPT algorithm. Therefore, these two algorithms are effective.

\section{Multi-objective Maintenance Scheduling Algorithm}

4.1 Multi-objective scheduling algorithm $\sum u_{i} / \sum_{i=1}^{n} c_{i}$ ( Moore-Hodgson/SPT scheduling algorithm)

Step 1: determine all combat units set that in need of repair, recorded as $G=\left\{t_{1}, t_{2}, \cdots, t_{m}\right\},(m \leq n)$;

Step 2: determine the malfunction equipment of all combat units, recorded as $G_{i}=\left\{t_{i j} \mid j \in\left\{1,2, \cdots, n_{i}\right\}\right\}$, including $i \in\{1,2, \cdots, m\}, \quad G_{i}$ is the i-th Combat units set.

Step 3: determine each combat unit's maintenance time $p_{i}(i=1,2, \cdots, m)$ and each fault equipment's maintenance time $p_{i j}\left(j=1,2, \cdots, n_{i}\right)$, meet the condition $p_{i}=\sum_{j=1}^{n_{i}} p_{i j}$.

Step 4: sort $p_{i}(i=1,2, \cdots, m)$ in non-increasing order, assuming that $p_{1} \geq p_{2} \geq \cdots \geq p_{m}$;

Step 4.1: if $p_{i}>p_{j}$, combat unit $i$ prior to unit $j$ in maintenance;

Step 4.2: if $p_{i}=p_{j}$, the shorter time of the combat unit's maintenance duration, the higher priority for repair, if some combat unit delay, select the former unit with the longest maintenance time and sort it to the last;

Step 5: using SPT scheduling algorithm for malfunction equipment $t_{i j} \in G_{i}, \quad j \in\left\{1,2, \cdots, n_{i}\right\}$, which in the same combat unit $t_{i}, \quad(i=1,2, \cdots, m)$.

Step 5.1: sorting the fault equipment's maintenance time according to ascending order, assuming $p_{i 1} \geq p_{i 2} \geq \cdots \geq p_{i m_{i}}$ (there are $m_{i}$ malfunction equipment in unit $i, m_{i} \leq n_{i}$ );

Step 5.2: according to the maintenance time from small to large order for maintenance.

4.2 Multi-objective scheduling algorithm $\sum u_{i} / \sum_{i=1}^{n} w_{i} c_{i}$ ( Moore-Hodgson/WSPT scheduling algorithm)

Step 1: determine the combat unit's weight $w_{i}(i=1,2, \cdots, m)$ and all combat units set that in need of repair, recorded as $G=\left\{t_{1}, t_{2}, \cdots, t_{m}\right\},(m \leq n)$;

Step 2: determine the malfunction equipment of all combat units, recorded as $G_{i}=\left\{t_{i j} \mid j \in\left\{1,2, \cdots, n_{i}\right\}\right\}$, including $i \in\{1,2, \cdots, m\}, G_{i}$ is the i-th Combat units set.

Step 3: determine each combat unit's maintenance time $p_{i}(i=1,2, \cdots, m)$ and each fault equipment's maintenance time $p_{i j}\left(j=1,2, \cdots, n_{i}\right)$, meet the condition $p_{i}=\sum_{j=1}^{n_{i}} p_{i j}$.

Step 4: calculated the ratio of combat unit's weight and maintenance time $\frac{w_{i}}{p_{i}},(i=1,2, \cdots, m)$, sort in non-increasing order, assuming that $\frac{w_{1}}{p_{1}} \geq \frac{w_{2}}{p_{2}} \geq \cdots \geq \frac{w_{m}}{p_{m}}$; 
Step 4.1: if $\frac{w_{i}}{p_{i}}>\frac{w_{j}}{p_{j}}$, combat unit $i$ prior to unit $j$ in maintenance;

Step 4.2: if $\frac{w_{i}}{p_{i}}=\frac{w_{j}}{p_{j}}$, the shorter time of the combat unit's maintenance duration, the higher priority for repair, if some combat unit delay, select the former unit with the longest maintenance time and sort it to the last;

Step 5: using SPT scheduling algorithm for malfunction equipment $t_{i j} \in G_{i}, \quad j \in\left\{1,2, \cdots, n_{i}\right\}$, which in the same combat unit $t_{i}, \quad(i=1,2, \cdots, m)$.

Step 5.1: sorting the fault equipment's maintenance time according to ascending order, assuming $p_{i 1} \geq p_{i 2} \geq \cdots \geq p_{i m_{i}}$ (there are $m_{i}$ malfunction equipment in unit $i, m_{i} \leq n_{i}$ );

Step 5.2: according to the maintenance time from small to large order for maintenance.

4.3 Example (with the objective function $\sum u_{i} / \sum_{i=1}^{n} w_{i} c_{i}$ as an example)

Assuming that there are six combat units, each of them is $A, B, C, D, E, F . A, B, C, D$ have been broke down. Duration, maintenance time and weight in Table 1.

Table 1 Combat Units

\begin{tabular}{|c|c|c|c|c|c|}
\hline $\begin{array}{c}\text { combat } \\
\text { unit }\end{array}$ & $\begin{array}{c}\text { malfunctio } \\
\mathrm{n} \\
\text { equipment }\end{array}$ & duration & $\begin{array}{c}\text { maintenance time of } \\
\text { malfunction equipment }\end{array}$ & $\begin{array}{c}\text { maintenance time } \\
\text { of combat unit }\end{array}$ & weight \\
\hline$A$ & $A_{1}, A_{2}, A_{3}$ & 25 & $6,9,5$ & 20 & 0.12 \\
\hline$B$ & $B_{1}, B_{2}, B_{3}$ & 25 & $8,3,1$ & 12 & 0.23 \\
\hline$C$ & $C_{1}, C_{2}$ & 8 & 7,3 & 10 & 0.25 \\
\hline$D$ & $D_{1}, D_{2}$ & 6 & 6,4 & 10 & 0.25 \\
\hline$E$ & & & & 0 & 0.13 \\
\hline$F$ & & & & 0 & 0.02 \\
\hline
\end{tabular}

the ratio of $\frac{w_{i}}{p_{i}}$ are, in order, 0.006, 0.019, 0.025, 0.025, sort according to descending order: $C$ equal with $D$, because of the duration of $D$ is shorter than $C$, so $B$ is in front of $A$, therefore, the optimal maintenance scheduling order is $D, C, B, A$. The maintenance sequence of combat unit $A$ is $A_{3}, A_{1}, A_{2}$; The maintenance sequence of combat unit $B$ is $B_{3}, B_{2}, B_{1}$; The maintenance sequence of combat unit $C$ is $C_{2}, C_{1}$, The maintenance sequence of combat unit $D$ is $D_{2}, D_{1}$.

\section{Conclusion}

For shortcomings in the current maintenance and support research, combined with the reality of modern warfare, we establish a fast scheduling model and give an effective algorithm. At last, an example shows that the model is easy to operate and practical. The direction of future research is multiple maintenance team in war.

\section{References}

[1] Maozhi Gan,jianshe Kang. Military Equipment Maintenance Engineering[M].Beijing: National Defence Industry Press,2005:1-2.

[2] Chen B.A Reviw of On-line Machine Scheduling: Algorithms and Cmpetitiveness[J]. Mathematical Theory and Application,1999,19(3):92-97.

[3] zhenyuan Wang. The dynamic maintenance tasks scheduling optimization method[J]. Journal of 
Mechanical Engineering, 2008,44(1):92-97.

[4] yu Zhu. Wartime equipment maintenance scheduling model based on maximum protection time[J]. Fire and Command Control,2009,34(1):11-14.

[5] jianjun Gao. study based on the combat effectiveness of the maintenance task scheduling model[C]// Annual Meeting of the Military Operations Research,2009.

[6] Li Z Y, Vairaktarkis G L. Complexity of Single Machine Hierarchical Scheduling: A Survey in: Parddolos, P.M. Complexity in Numerical Optimization[M]. New Jersey: World Scientific Publishing Company,1993:269-298.

[7] guochun Tang. Modern Sort On[M]. Shanghai: Shanghai Popular Science Press,2003.

[8] liqiang Jiang. Weapon system maintainability allocation planning methods research[J]. Systems Engineering and Electronics,2007,29(1):155-157. 